University of New Hampshire

University of New Hampshire Scholars' Repository

Space Science Center

Institute for the Study of Earth, Oceans, and

Space (EOS)

1993

\title{
COMPTEL observations of Cygnus X-1
}

\author{
Mark L. McConnell \\ University of New Hampshire - Main Campus, mark.mcconnell@unh.edu \\ A Connors \\ University of New Hampshire - Main Campus \\ D J. Forrest \\ University of New Hampshire - Main Campus \\ James M. Ryan \\ University of New Hampshire, James.Ryan@unh.edu \\ W Collmar \\ Max-Planck-Institut für extraterrestriche Physik
}

See next page for additional authors

Follow this and additional works at: https://scholars.unh.edu/ssc

Part of the Astrophysics and Astronomy Commons

\section{Recommended Citation}

COMPTEL observations of Cygnus X-1 McConnell, M. and Connors, A. and Forrest, D. and Ryan, J. and Collmar, W. and Diehl, R. and Schönfelder, V. and Steinle, H. and Strong, A. and Bloemen, H. and van Dijk, R. and Hermsen, W. and Kuiper, L. and Swanenburg, B. and Winkler, C., AIP Conference Proceedings, 280, 335-339 (1993), DOI:http://dx.doi.org/10.1063/1.44142

This Conference Proceeding is brought to you for free and open access by the Institute for the Study of Earth, Oceans, and Space (EOS) at University of New Hampshire Scholars' Repository. It has been accepted for inclusion in Space Science Center by an authorized administrator of University of New Hampshire Scholars' Repository. For more information, please contact Scholarly.Communication@unh.edu. 


\section{Authors}

Mark L. McConnell, A Connors, D J. Forrest, James M. Ryan, W Collmar, R Diehl, V Schonfelder, H Steinle, A W. Strong, H Bloemen, R Van Dijk, W Hermsen, L Kuiper, B Swanenburg, and C Winkler 


\section{AIP | proceedings}

\section{COMPTEL observations of Cygnus X1}

M. McConnell, A. Connors, D. Forrest, J. Ryan, W. Collmar, R. Diehl, V.

Schönfelder, H. Steinle, A. Strong, H. Bloemen, R. van Dijk, W. Hermsen, L.

Kuiper, B. Swanenburg, and C. Winkler

Citation: AIP Conference Proceedings 280, 335 (1993); doi: 10.1063/1.44142

View online: http://dx.doi.org/10.1063/1.44142

View Table of Contents:

http://scitation.aip.org/content/aip/proceeding/aipcp/280?ver=pdfcov

Published by the AIP Publishing

\section{Articles you may be interested in}

COMPTEL measurements of $1.809 \mathrm{MeV}$ gammaray line emission from the Galactic plane

AIP Conf. Proc. 280, 40 (1993); 10.1063/1.44309

OSSE spectral observations of GC 339-4 and Cyg X-1

AIP Conf. Proc. 280, 345 (1993); 10.1063/1.44295

Longterm temporal and spectral variation of Cygnus X1 observed by BATSE AIP Conf. Proc. 280, 340 (1993); 10.1063/1.44294

Gammaray burst studies by COMPTEL during its first year of operation AIP Conf. Proc. 280, 845 (1993); 10.1063/1.44232

Earth occultation measurements of galactic hard Xray/gammaray sources: A survey of BATSE results

AIP Conf. Proc. 280, 314 (1993); 10.1063/1.44138 


\section{McConnell, A. Connors, D. Forrest, J. Ryan \\ Space Science Center, University of New Hampshire, Durham, NH 03824 \\ W. Collmar, R. Diehl, V. Schönfelder, H. Steinle, A. Strong Max Planck Institute for Extraterrestrial Physics, 8046 Garching, Federal Republic of Germany}

H. Bloemen, R. van Dijk*, W. Hermsen, L. Kuiper, B. Swanenburg SRON-Leiden, 2300 RA Leiden, The Netherlands

C. Winkler

Astrophysics Division, European Space Research and Technology Center, 2200 AG Noordwijk, The Netherlands

\section{ABSTRACT}

We report on the latest results from an analysis of data on Cygnus $\mathrm{x}-1$ as collected by the COMPTEL experiment on the compton Gamma-Ray observatory (CGRO). COMPTEL detected the source around 1 $\mathrm{MeV}$ during two separate one-week observations in 1991. The measured flux levels indicate variability on a time scale of weeks. The results also indicate flux levels which are well below the levels reported by some investigators for an excess of emission in the energy range $\geq 1 \mathrm{MeV}$.

\section{INTRODUCTION}

The COMPTEL experiment on the Compton Gamma-Ray Observatory is designed to measure gamma-radiation in the energy range from $0.75-30$ MeV. This is a particularly interesting energy range for the blackhole candidate Cygnus $x-1$. Various observers have reported detections of excess emission at energies around $1 \mathrm{MeV}$ beyond that which would be expected based on an extrapolation of the hard $x$-ray spectrum $1,2,3$. The reported flux levels are more than two orders of magnitude above the COMPTEL detection threshold, suggesting that COMPTEL is capable of studying such emission in detail. During the first year of CGRO orbital operations, Cygnus $x-1$ was within the COMPTEL field-of-view on two separate occasions. The first observation took place in early June of 1991. The second observation took place some 1-1/2 months later, in August, 1991. Cygnus $x-1$ is clearly seen in data collected during both observations, with a weaker signal detected during the first observation. Here we shall present the latest results from the analysis of these data along with a comparison between the COMPTEL data and earlier measurements at these energies.

* Astronomical Institute "Anton Pannekoek", University of Amsterdam 


\section{OBSERVATIONS}

During the first year of orbital operations, the COMPTEL experiment observed the Cygnus region on two separate occasions. The first observation, which was part of the planned 15-month sky survey, took place from May 30 to June 8, 1991 (Viewing Period 2.0).

Although planned as a full two-week exposure, this observation was interrupted after only nine days due to the declaration of a solar target-of-opportunity for CGRO. A second opportunity to observe the Cygnus region came only 1-1/2 months later, when another target-ofopportunity was declared to observe Cygnus $x-3$ (which had recently exhibited an intense outburst of radio emission). This observation (Viewing Period 7.0) lasted from August 8 to August 15, 1991. The net result was a total exposure which exceeded the standard 14-day observation. More importantly, these data provided an opportunity to study time variability during the intervening $1-1 / 2$ month time interval.

During the early phases of the GRO mission, the COMPTEL instrument was not operating at full capacity. This was due primarily to out-gassing effects which were noted in certain detector modules. For this reason, the affected modules remained turned of for most of the early part of the mission. During V.P. 2.0, the instrument configuration changed frequently. For the purposes of the present anlysis, only eight days of data were used. Three of the 14 D2 modules were not active during this period; this amounts to a reduction in overall COMPTEL efficiency by $21 \%$. During V.P. 7.0, one $D 1$ and one D2 module were inactive; this is equivalent to a reduction of about $20 \%$ in the overall COMPTEL efficiency.

\section{ANALYSIS}

The analysis of the COMPTEL imaging data for these two observation periods has been performed using the maximum likelihood method. 5 This method provides quantitative information regarding the source location and flux. For the present analysis, no independent estimate of the background (predominantly instrumental in origin) was available for the maximum likelihood analysis. Therefore, an estimate of the background was derived directly from the source data by an averaging technique which suppresses point-source signals, but preserves the general background structure.

For the present analysis, the response information comes from a COMPTEL simulation model. The simulation model is based on the CERN GEANT code and is used to simulate a large number of events which can then be used to define the PSF of the COMPTEL instrument. The present results are somewhat limited in terms of the number of simulated events which have been used to generate the PSFs. Therefore, it can be expected that the present results may be somewhat modified as the PSF staisistics are improved with additional simulations. 
It is also important to ensure that the PSF be defined for a spectrum which accurately represents the spectrum of the observed source. For the results described here, the PSEs were generated for an input wien-type spectrum, which represents the high energy limit of the Sunyaev-Titarchuk inverse-Compton spectrum. 6 In addition to the normalization, this spectrum has only a single parameter - the electron temperature (kT) of the accreting plasma. Given the relatively high energy threshold of the COMPTEL data, the resulting observations are relatively insensitive to the Compton scattering optical depth.

\section{RESULTS}

In Figure 1 we show a maximum likelihood map of the Cygnus region as derived from the $0.75-1.0 \mathrm{MeV}$ data collected during V.P. 7.0. Cygnus $x-1$ dominates the image. There is no apparent signature in the direction of Cygnus $x-3$; this is important to note, given that V.P. 7.0 was also the Cygnus $x-3$ target-of-opportunity. Upper limits for Cygnus $x-3$ have not yet been derived from these data.

Positive flux measurements are found for both observation periods in the range of $0.75-3.0 \mathrm{MeV}$. Only upper limits are available at higher energies. The flux levels found in both the $0.75-1.0 \mathrm{MeV}$ band and the $1.0-3.0 \mathrm{MeV}$ band are about a factor of two lower during V.P. 2.0 than those in V.P. 7.0.

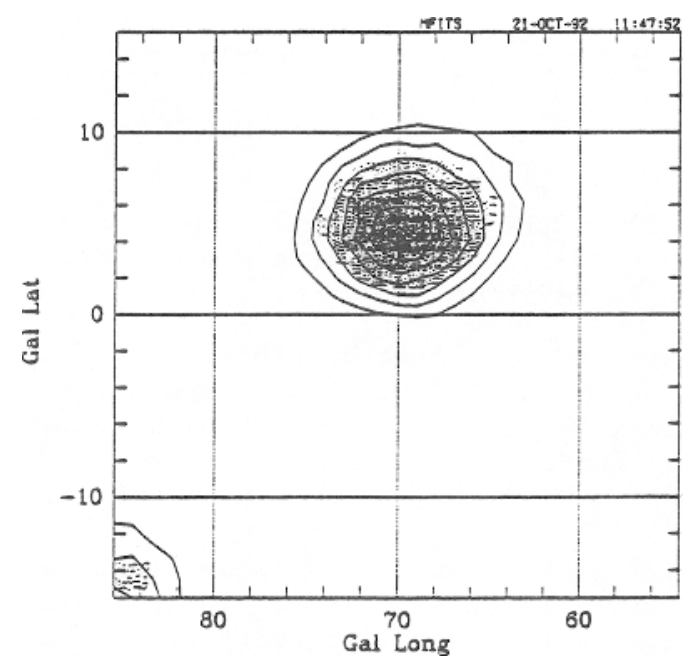

Fig. 1. Maximum likelihood image of the Cygnus region for the $0.75-1.0 \mathrm{MeV}$ data collected during viewing period 7.0. The response from Cygnus $x-1$ (at $I=71.3^{\circ}, b=3.1^{\circ}$ ) stands out clearly whereas there appears to be no evidence for emission from Cygnus $x-3$ (at $1=79.9^{\circ}$, $b=0.7^{\circ}$ ). 
For V.P. 7.0, we have crudely estimated the electron plasma temperature (kT) by comparing the measured flux ratio in the two lowest energy bands with that expected for the wien spectrum used in the corresponding PSFs. It is found that the derived flux ratio agrees fairly well with that predicted for an electron temperature in

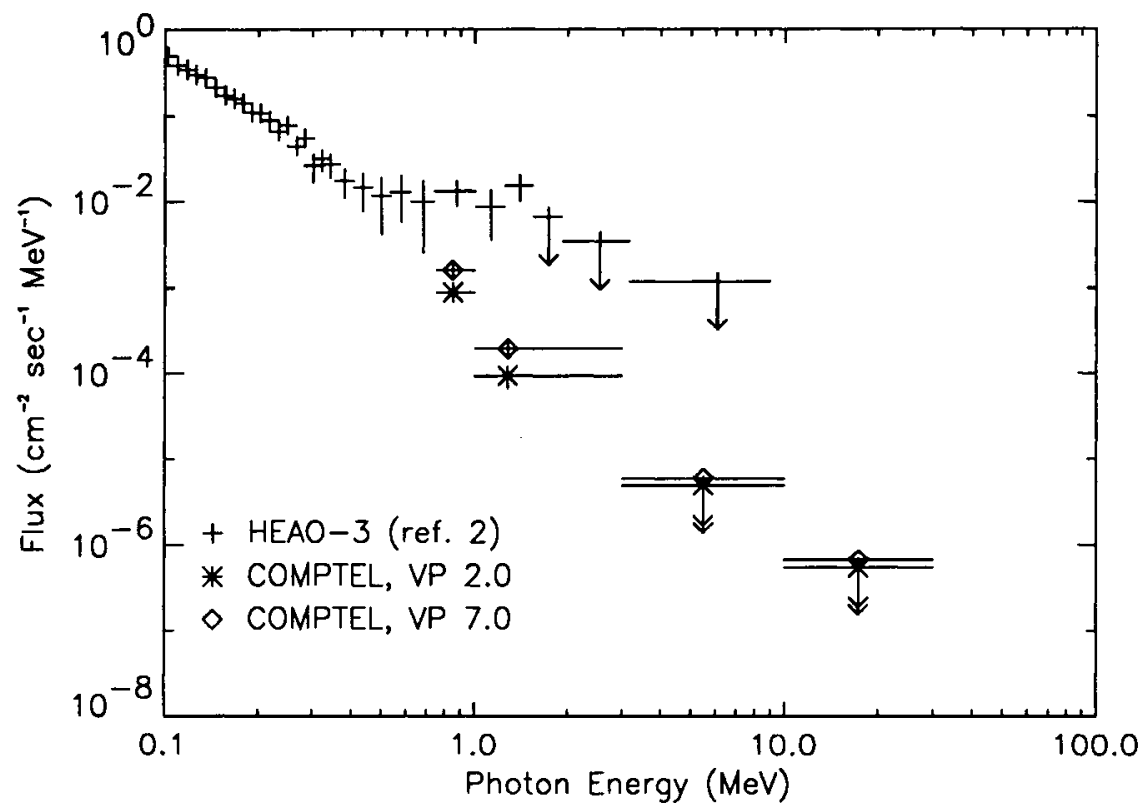

Fig. 2. The COMPTEL spectra compared with the $\gamma_{1}$ spectrum of HEAO-3. COMPTEL data below $3 \mathrm{MeV}$ are plotted based on an $80 \mathrm{keV}$ wien spectrum.

the range of $80-100 \mathrm{keV}$. This is consistent with many previous measurements of the Cygnus $\mathrm{x}-1$ electron temperature at hard $\mathrm{x}$-ray energies. 7 Figure 2 shows the two COMPTEL measurements relative to the $\gamma_{1}$ spectrum as measured by Ling et al. (1987). The COMPTEL measurements are more than one order of magnitude below the HEAO-3 data.

Several relevant balloon observations are shown in Figure 3 along with the COMPTEL results for V.P. 7.0 (the higher of the two COMPTEL flux measurements). As with the HEAO-3 data, the COMPTEL data points fall far below some of these data. The UCR Compton telescope upper limits are, however, compatible with the COMPTEL results.

\section{DISCUSSION AND SUMMARY}

The present COMPTEL results are interesting in that they permit a high-sensitivity measurement of the Cygnus $x-1$ spectrum to energies 


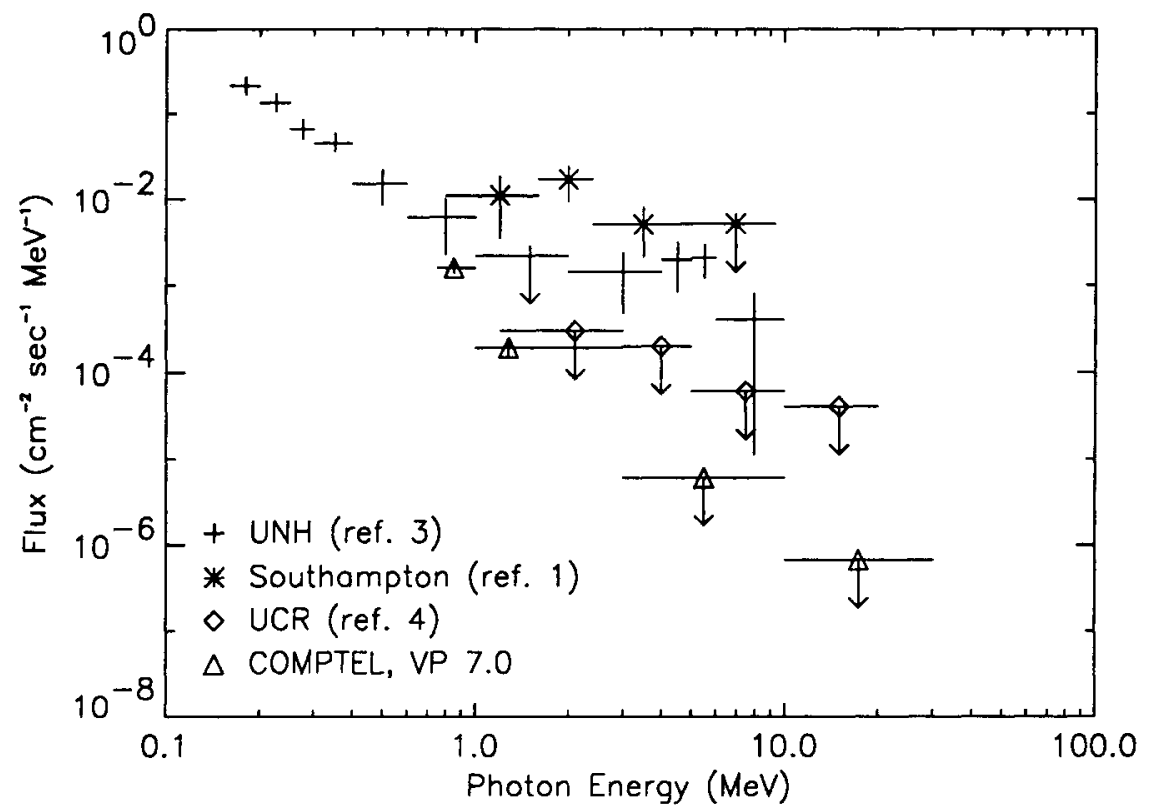

Fig. 3. The COMPTEL spectra compared with various balloon measurements. COMPTEL data below 3 MeV are plotted based on an $80 \mathrm{keV}$ wien spectrum.

above $1 \mathrm{MeV}$. These data show no evidence for any excess emission near $1 \mathrm{MeV}$ as has often been reported in the past. It is interesting to note that during Viewing Period 2.0, the OSSE spectral data indicate that the hard $x$-ray flux was in a relatively low state. ${ }^{8}$ More specifically, it was in a level comparable to that of the $\gamma_{1}$ state as defined by the HEAO- 3 measurements ${ }^{2}$. The excess of emission near $1 \mathrm{MeV}$ observed by HEAO-3 was found during a correspondingly low level of hard $x$-ray flux. The fact that the COMPTEL results show no evidence for any excess emission suggests that the low hard $x$-ray flux may be a necessary condition for MeV emission, but it is certainly not a sufficient condition.

\section{REFERENCES}

1. R. Baker et al., Nature 245, 18 (1973).

2. J. Ling et al., Ap. J. (Letters) 321, L117 (1987).

3. M. McConnell et al., Ap. J. 343, 317 (1989).

4. R. White et al., Nature 284, 608 (1980)

5. H. de Boer et al., in "Data Analysis in Astronomy IV", ed. V. di Gesù et al. (Plenum Press, 1992), p. 241.

6. R. Sunyaev and L. Titarchuk, Astron. Astrophys., in press (1992).

7. A. Owens and M. McConnell, Comments in Astrophys., in press (1992).

8. W. Johnson et al., Astron. Astrophys., in press (1992) 